\title{
Cooling ultracold bosons in optical lattices by spectral transform
}

\author{
David L. Feder \\ Department of Physics and Astronomy and Institute for Quantum Information Science, \\ University of Calgary, Calgary, Alberta, Canada T2N 1N4
}

(Dated: November 3, 2018)

\begin{abstract}
It is shown theoretically how to directly obtain the energy distribution of a weakly interacting gas of bosons confined in an optical lattice in the tight-binding limit. This is accomplished by adding a linear potential to a suitably prepared lattice, and allowing the gas to evolve under the influence of the total potential. After a prescribed time, a spectral transform is effected where each (highly non-local) energy state is transformed into a distinct site of the lattice, thus allowing the energy distribution to be (non-destructively) imaged in real space. Evolving for twice the time returns the atoms to their initial state. The results suggest efficient methods to both measure the temperature in situ, as well as to cool atoms within the lattice: after applying the spectral transform one simply needs to remove atoms from all but a few lattice sites. Using exact numerical calculations, the effects of interactions and errors in the application of the lattice are examined.

PACS numbers: 37.10.Jk, 37.10.De, 67.85.-d
\end{abstract}

\section{INTRODUCTION}

Despite the strong interest in the properties of ultracold bosons confined in optical lattices [1, 2, 3, 3, 4, 5], the accurate determination and efficient control of the temperature remains a persistent problem. The initial loading of the atoms into optical lattices can cause significant heating if it is not performed adiabatically [6] which becomes increasing difficult as the lattice deepens [7]. Likewise, adiabatic changes to the lattice potential itself can both increase or decrease the temperature [8, 9, 10]. Instrinsic problems such as laser motion [11], atomic motion [12], or impurities [13, 14] can also adversely affect the temperature. The inability to achieve very low temperatures in optical lattices is one of the primary obstacles to achieving and controlling interesting quantum states in these systems [15, 16, 17].

There is currently no efficient way to directly measure the temperature of the bosons when quantum mechanical tunneling within the lattice is appreciable. This weakly correlated regime is applicable for sufficiently shallow lattices, weak interactions, or large numbers of atoms at each site [18]. The standard method is to release the atoms from the trap, and is fully destructive [19]. The shadow-image of the fully expanded cloud then corresponds approximately to the momentum distribution in the trap, though the presence of atomic interactions modifies this simple picture somewhat [20, 21]. In contrast, in the weak tunneling (strongly correlated) limit there are several proposed methods for inferring the temperature, including measuring the density distribution of doubly occupied sites 22] and the atomic number fluctuations [23].

Unfortunately, directly cooling weakly correlated atoms in an optical lattice is not straightforward. Only one procedure has been proposed, based on a combination of sophisticated quantum optics and many body effects [24]. In contrast, under conditions of weak tunneling several techniques have been proposed [25]. The standard evaporative cooling technique for bosons in harmonic traps [26] is predicated on the correspondence between the energy and spatial extent of the single-particle quantum states. Atoms far from the trap center tend to have a higher energy, so their gradual removal combined with rethermalization can rapidly cool the particles. This approach unfortunately cannot be applied to optical lattices even if they are shallow, however, because all singleparticle energy states span the length of the lattice.

This work proposes that optical lattices can be suitably prepared in order to allow for the direct measurement of the temperature of a weakly correlated Bose gas, and also to efficiently cool it. The main idea is that if one could vary the depth of the optical lattice quadratically along its length, then to an excellent approximation the low-energy single-particle states of the system are the discrete-space analogs of the harmonic oscillator Hermite polynomials, known as Krawtchouk functions [27, 28]. As discussed in detail below, this could be accomplished using at least two distinct and readily available methods. First, one could change the focal length of one of the optical lattice lasers, so that the intensity maxima of the two beams are displaced relative to one another. Second, one could add an additional optical lattice at a small angle relative to the first. In both cases, if the temperature is already relatively cold, then an in-situ non-destructive image will reveal a bimodal density distribution much like those found for bosons in harmonic traps [29]. Evaporative cooling can then be performed directly.

For larger temperatures where the tight-binding band is close to full [30] and all single-particle states are strongly overlapping, the energy distribution of the gas can be obtained by performing a full spectral transformation. This is accomplished by adding a small linear potential 31, and allowing the system to evolve in time. Single-particle states with low energies will evolve with high probability into the occupation of lattice sites on the left, while high-energy states will migrate to sites on the right. Thus, after a prescribed evolution time the 
spatial profile of the atomic density yields the initial energy distribution. Further cooling can then be effected simply by preferentially removing atoms toward the right by imaging with a resonant laser. Time-evolving further performs the reverse transformation, mapping the singleparticle states back to their original representation.

While this approach is exact in the limit of noninteracting particles (and therefore much in the spirit of evaporative cooling ideas in harmonic traps) and only for a particular choice of tight-binding Hamiltonian, it is robust with respect to both atomic interactions and to errors in the application of the optical lattice, as shown in detail toward the end of this manuscript. It is equally applicable to one, two, or three dimensional systems.

The manuscript is organized as follows. The basic formalism for atoms in optical lattices and the tightbinding approximation are reviewed in Sec. III The conditions under which it is possible in principle to perform a spectral transform are discussed in Section III. The Krawtchouk functions are introduced in Section [V] and the condition under which these may effect a spectral transform is also shown. The robustness of the results to the presence of interactions and approximations to the optical lattice potential are explored in Section V and the results are discussed and summarized in Section VI.

\section{ATOMS IN OPTICAL LATTICES}

Optical lattices are standing waves formed by overlapping, focused lasers. In the case of a one-dimensional (1D) lattice, the lasers can be considered to be counterpropagating. Assuming that the lattice is oriented along the axial $\hat{z}$ direction and is axisymmetric, the lattice potential is 32]

$$
V_{1}(r, z)=-V(z) \exp \left(-r^{2} / r_{0}^{2}\right) \sin ^{2}(k z),
$$

where $r$ is the transverse coordinate, $V(z)>0$ is the lattice depth that may depend on the axial coordinate, $r_{0}$ is the transverse beam radius, and $k=2 \pi / \lambda$ is the wavenumber of the light with wavelength $\lambda$. This expression assumes that the confinement is strongest (the potential is most negative) at points of largest laser intensity (i.e. when the frequency $c / \lambda$ of the laser is smaller than that of the main atomic resonance, known as reddetuning [26]). Allowing for the possibility of an additional external potential $V_{e}(z)$ aligned along the lattice axis, the Hamiltonian for interacting bosons is

$$
\begin{aligned}
H & =\int d^{3} r \psi^{\dagger}(r, z)\left[-\frac{\hbar^{2}}{2 m} \nabla^{2}+V_{1}(r, z)+V_{\mathrm{e}}(z)\right] \psi(r, z) \\
& +\frac{g}{2} \int d^{3} r d^{3} r^{\prime} \psi^{\dagger}(r, z) \psi^{\dagger}\left(r^{\prime}, z^{\prime}\right) \psi\left(r^{\prime}, z^{\prime}\right) \psi(r, z),
\end{aligned}
$$

where $\psi^{\dagger}(r, z)$ creates a boson field at position $(r, z)$, $g=4 \pi \hbar^{2} a / m$ is the two-body coupling constant [26], and $a$ is the $s$-wave scattering length. For ${ }^{87} \mathrm{Rb}$, for example, the value of the scattering length is $a \approx 100 a_{0}=$
$5.29 \mathrm{~nm}$ [33], with $a_{0}$ the Bohr radius. It is convenient to express all energies in terms of the atomic recoil energy $E_{R} \equiv \hbar^{2} k^{2} / 2 m=2 \pi^{2} \hbar^{2} / m \lambda^{2}$. The coupling constant is then $g=\left(2 \lambda^{2} a / \pi\right) E_{R}$.

In the limit of very strong transverse confinement [34], all of the boson fields will occupy the lowest-energy eigenstate of the confining potential in the transverse direction. Neglecting the possible $z$-dependence of the lattice potential for the time being, one obtains $V_{1}(r) \approx$ $-V\left(1-r^{2} / r_{0}^{2}\right)=-V+V r^{2} / r_{0}^{2}$ which is approximately harmonic with effective frequency $\omega=\sqrt{2 V / m r_{0}^{2}}$ and harmonic oscillator length $\ell=\sqrt{\hbar / m \omega}$. Making the substitution $\psi^{\dagger}(r, z) \approx \sqrt{2 / \pi \ell^{2}} \exp \left(-r^{2} / 2 \ell^{2}\right) \psi^{\dagger}(z)$ and integrating over the radial coordinate gives a rescaled coupling constant $g_{1 \mathrm{D}}=g / \pi \ell^{2}=4 \pi a \hbar \omega=4 \sqrt{\bar{V}}\left(a \lambda / r_{0}\right) E_{R}$, where $\bar{V}=V / E_{R}$ is the lattice depth in recoil energies.

Focusing the optical lattice lasers generally leads to an axially-dependent lattice depth $V(z)$ that varies over the Rayleigh length. As a result, the lattice depth is usually largest at the center and exponentially decreasing on either side $V(z)=V_{0} \exp \left(-z^{2} / z_{0}^{2}\right)$, though with a very long beam waist $z_{0}$. Beam waists for optical potentials as long as $125 \mu \mathrm{m}$ [35] and for optical lattices as small as $60 \mu \mathrm{m}$ [36] have been employed in recent experiments.

An excellent description of ultracold atoms in optical lattice potentials of the moderate depth discussed above is provided by the Bose-Hubbard Hamiltonian 37]. The Bose field operators are expanded $\psi(z)=\sum_{i} b_{i} w\left(z-z_{i}\right)$ in a basis of Wannier functions $w\left(z-z_{i}\right)$ that are highly localized near a lattice site $i$. Assuming that the energies related to the particle dynamics and the temperature $k_{B} T$ are both small compared with the bandgap, one obtains the Bose-Hubbard Hamiltonian [38]:

$$
H=-\sum_{\langle i j\rangle} J_{i, j} b_{i}^{\dagger} b_{j}+\frac{U}{2} \sum_{i} n_{i}\left(n_{i}-1\right)+\sum_{i} \varepsilon_{i} b_{i}^{\dagger} b_{i},
$$

where $\langle i j\rangle$ denotes a sum over nearest neighbours (i.e. $j=i \pm 1), b_{i}^{\dagger}$ creates a boson at site $i$, and $n_{i}=b_{i}^{\dagger} b_{i}$ is the density operator for site $i$. For sufficiently deep lattices $V_{0} \gtrsim 5 E_{R}$, one need only consider tunneling of atoms between nearest-neighbor sites and interactions of atoms within the same lattice site; the amplitudes for hopping between next-nearest-neighbor sites and the strength of interactions between atoms on neighboring sites are smaller by at least an order of magnitude, respectively [37].

If the external potential $V_{e}(z)$ is slowly varying over the lattice spacing, the hopping coefficient is

$$
J_{i, j}=\int d z w^{*}\left(z-z_{i}\right)\left[-\frac{\hbar^{2}}{2 m} \nabla^{2}+V_{\mathrm{l}}(z)\right] w\left(z-z_{j}\right) .
$$

In the very deep lattice limit where the value of the lattice depth at site $i$ (in recoil energies) is large $\bar{V}_{i} \equiv$ $V_{i} / E_{R} \gg 1$, the Wannier functions can be approximated by the ground-state Gaussian wavefunctions for each separate well $w(z) \approx e^{-z^{2} / 2 d^{2}} / \sqrt{d \sqrt{\pi}}$ with $d=\left(k \bar{V}_{i}^{1 / 4}\right)^{-1}$. 
Then the hopping amplitudes (4) are given by $J_{i, j} / E_{R}=$ $\left(\pi^{2} / 4\right) \bar{V}_{i} \exp \left(-\pi^{2} \sqrt{\bar{V}_{i}} / 4\right)$. A better approximation for the Wannier functions instead gives [5, 22]

$$
\frac{J_{i, j}}{E_{R}} \approx \sqrt{\frac{16}{\pi}} \bar{V}_{i}^{3 / 4} \exp \left(-2 \sqrt{\bar{V}_{i}}\right) .
$$

Meanwhile, the on-site interaction term is

$$
U=g_{1 \mathrm{D}} \int d z|w(z)|^{4} .
$$

Again approximating the Wannier functions by harmonic oscillator functions, one obtains

$$
\frac{U}{E_{R}}=\sqrt{\frac{8}{\pi}} \frac{a \lambda k}{r_{0}} \bar{V}^{3 / 4}=\sqrt{32 \pi} \frac{a}{r_{0}} \bar{V}^{3 / 4} .
$$

Finally, the site-dependent local energy is

$\varepsilon_{i}=\int d z w^{*}\left(z-z_{i}\right)\left[-\frac{\hbar^{2}}{2 m} \nabla^{2}+V_{\mathrm{l}}(z)+V_{\mathrm{e}}(z)\right] w\left(z-z_{i}\right)$.

This latter term is a constant energy offset for homogeneous lattice potentials and no exernal potential, and is usually dropped. In the present case, however, the lattice amplitude will vary slowly over the lattice spacing $\lambda / 2$. This will give rise to a spatial variation of $\varepsilon_{i}$, which for large lattices is equivalent to a smoothly varying external potential even in the absence of any $V_{\mathrm{e}}(z)$. Again using the local harmonic oscillator solutions for the Wannier functions, one obtains $\varepsilon_{i} / E_{R} \approx \sqrt{\bar{V}_{i}}$.

The onset of strong correlations for bosons in quasi1D optical lattices with an average of $\langle n\rangle \gg 1$ atoms per site occurs when $U / J>2.2\langle n\rangle$ [5, 39, 40]. Combining Eqs. (5) and (7), one obtains the criterion

$$
\sqrt{2} \pi \frac{a}{r_{0}} e^{2 \sqrt{\bar{V}}}>2.2\langle n\rangle .
$$

The value of $r_{0}$ is set by imposing the quasi-1D criterion $\hbar \omega \gtrsim \bar{V} E_{R}$, or $r_{0} / \lambda \ll(\pi \sqrt{\bar{V}})^{-1}$. This condition is in fact much more restrictive than necessary because at low temperatures $T \propto J$ (for example, the BEC transition temperature for a $3 \mathrm{D}$ lattice is $k_{B} T_{c}=5.59 \mathrm{~J}[5]$ ) only the first band is occupied, and the bandwidth in the 1D tight binding limit is $w=2 J \ll V$. With $\hbar \omega=\bar{V} E_{R}$, $a=5.29 \mathrm{~nm}$, and $\lambda=800 \mathrm{~nm}$, and a typical value $\langle n\rangle=$ 1000 one obtains $\bar{V}_{\max } \lesssim 16$ and $r_{0} \approx 32 \mathrm{~nm}$ to ensure that the atoms remain in the weakly correlated regime. This transverse length scale corresponds to a confining frequency $\omega / 2 \pi \approx 100 \mathrm{kHz}$, a typical value in current experiments [5]. With $\langle n\rangle=10000$ instead, one obtains $\bar{V}_{\max } \approx 25$.

Far in the weakly correlated regime, the amplitude for nearest-neighbor tunneling from site to site tends to be larger (and therefore more important) than the strength of particle interactions. As a first approximation, we may therefore set $U=0$; the consequences of $U \neq 0$ will be explored in Sec. V Furthermore, it will be assumed that $V_{e}(z)$ can be chosen in such a way to ensure that $\varepsilon_{i}$ is approximately constant, so that it can be ignored (this assumption will be further justified in Sec. VA . The resulting non-interacting tight-binding Hamiltonian can then be written

$$
H_{0}=-\sum_{\langle i j\rangle} J_{i, j}|i\rangle\langle j|
$$

where the site state kets are defined by $|i\rangle \equiv b_{i}^{\dagger}|\mathbf{0}\rangle$ and $|\mathbf{0}\rangle$ is the vacuum state.

In principle, the position indices $i$ and $j$ are unrestricted, but in practice the lattice has a finite number of occupied sites. This could arise naturally because of the inherent spatial variation of the lattice potential discussed above, in which larger number of atoms will occupy the central region where the potential is deepest. To enforce occupation of a specific part of the lattice, one can apply 'end-cap' potentials formed from tightly focused blue-detuned lasers [34, 35]. In this case, one can effectively enforce the 'hard-wall' boundary conditions $i \in\{0, N\}$ with $j=i \pm 1$ when $0<i<N$, but $j=1$ only for $i=0$ and $j=N-1$ only when $i=N$.

\section{SPECTRAL TRANSFORM}

\section{A. Introduction}

As is well-known from elementary quantum mechanics, an arbitrary quantum state $|\psi(j)\rangle$ defined on $N+1$ discrete sites labelled by index $j$ can always be expressed in terms of an arbitrary basis of dimension $N+1$

$$
|\psi(j)\rangle=\sum_{n=0}^{N} c_{n}\left|\varphi_{n}(j)\right\rangle,
$$

where the $c_{n}$ are generally complex coefficients. The set of states $\left\{\left|\varphi_{n}(j)\right\rangle, n \in 0, N\right\}$ is assumed to be both orthonormal

$$
\sum_{j=0}^{N}\left\langle\varphi_{m}(j) \mid \varphi_{n}(j)\right\rangle \equiv\left\langle\varphi_{m} \mid \varphi_{n}\right\rangle=\delta_{m, n}
$$

and complete

$$
\sum_{n}\left\langle\varphi_{n}(j) \mid \varphi_{n}(k)\right\rangle=\delta_{j, k},
$$

where the Kronecker delta is defined as $\delta_{j, k}=1$ only if $j=k$ and is zero otherwise. Multiplying both sides of Eq. (11) on the left by $\left\langle\varphi_{m}(j)\right|$ and summing over $j$, one obtains the coefficients using (12):

$$
c_{n}=\sum_{j}\left\langle\varphi_{n}(j) \mid \psi(j)\right\rangle \equiv\left\langle\varphi_{n} \mid \psi\right\rangle .
$$


One could have obtained this directly simply by inserting the complete set, $|\psi\rangle=\sum_{n}\left|\varphi_{n}\right\rangle\left\langle\varphi_{n} \mid \psi\right\rangle$.

The relevance of the above discussion to the present work is that the coefficients $c_{n}$ can be considered as the representation of the original wavefunction in the 'spectral basis' rather than the original site basis. While the elements of the original wavefunction in the site basis are

$$
\langle j \mid \psi\rangle \equiv \psi(j)=\sum_{n} c_{n}\left\langle j \mid \varphi_{n}\right\rangle=\sum_{n} c_{n} \varphi_{n}(j),
$$

the elements of the same wavefunction in the spectral basis are

$$
\left\langle\varphi_{n} \mid \psi\right\rangle \equiv c_{n}=\sum_{j}\left\langle\varphi_{n} \mid j\right\rangle\langle j \mid \psi\rangle=\sum_{j} \psi(j) \varphi_{n}^{*}(j) .
$$

Eqs (15) and (16) are transforms of each other through the basis functions $\varphi_{n}(j)$; the second can be immediately obtained from the first from their orthonormality (12), and the converse follows from their completeness (13). These generalize the Fourier transforms that are specific to the regular lattice case, in which case $\varphi_{n}(j) \propto \exp [i 2 \pi n j /(N+1)]$.

The purpose of the current work is to show that the coefficients $c_{n}$ in Eq. (11) can be obtained directly in experiments without having to explicitly carry out the transformation (16). In particular, if one could implement the spectral transform

$$
\left|\varphi_{n}\right\rangle \rightarrow e^{i \phi_{n}}|n\rangle
$$

with arbitrary phases $\phi_{n}$, then all particle densities would be transformed as $|\psi(j)|^{2} \rightarrow\left|c_{j}\right|^{2}$ :

$$
\begin{aligned}
|\psi(j)|^{2} & =\langle j \mid \psi\rangle\langle\psi \mid j\rangle=\sum_{n} c_{n}\left\langle j \mid \varphi_{n}\right\rangle \sum_{m} c_{m}^{*}\left\langle\varphi_{m} \mid j\right\rangle \\
& \rightarrow \sum_{m, n} c_{n} c_{m}^{*} e^{i\left(\phi_{n}-\phi_{m}\right)}\langle j \mid n\rangle\langle m \mid j\rangle=\left|c_{j}\right|^{2} .
\end{aligned}
$$

Thus after the spectral transformation, the magnitude of the coefficients in the expansion (11) would be obtained simply from the values of the density at each lattice point.

\section{B. Transform by Hamiltonian Evolution}

Consider a single particle hopping on $N+1$ sites of a 1D lattice with hard-wall boundary conditions, as defined by the non-interacting tight-binding Hamiltonian (10) with $J_{i, j}=J_{j, i}$ and $J_{-1,0}=J_{N, N+1}=0$. This Hamiltonian has $N+1$ eigenvectors $\left|v_{j}\right\rangle$ with eigenvalues $\epsilon_{j}$. Now consider the perturbed Hamiltonian $H=H_{0}+H_{1}$ with completely local (i.e. on-site) potential

$$
H_{1}=\sum_{j=0}^{N} V_{j}|j\rangle\langle j|
$$

with real $V_{i}$. (Note that the $V_{i}$ is an external local potential that is applied in addition to the $V_{e}(z)$ chosen above to ensure constant $\varepsilon_{i}$ ). The objective is to determine the $V_{j}$ for a given choice of $J_{i, j}$ such that time-evolution governed by the full Hamiltonian $H$ effects the desired spectral transformation (17), but now with $\left|v_{n}\right\rangle$ taking the place of the $\left|\phi_{n}\right\rangle$ above:

$$
\exp \left(-i H t_{s} / \hbar\right)\left|v_{j}\right\rangle=e^{i \phi_{j}}|j\rangle
$$

where $t_{s}$ is some fixed time satisfying the condition for any $j \in\{0, N\}$ and $\phi_{j}$ is a possible arbitrary phase.

It is insightful to write the desired unitary $U=$ $\exp \left(-i H t_{s} / \hbar\right)$ in its spectral decomposition

$$
U=\sum_{k=0}^{N} \exp \left(-i \epsilon_{k}^{\prime} t_{s} / \hbar\right)\left|v_{k}^{\prime}\right\rangle\left\langle v_{k}^{\prime}\right|,
$$

where $\epsilon_{k}^{\prime}$ and $\left|v_{k}^{\prime}\right\rangle$ are the eigenvalues and eigenvectors of $H$, respectively. The transform (20) can then be written

$$
U\left|v_{j}\right\rangle=\sum_{k=0}^{N} \exp \left(-i \epsilon_{k}^{\prime} t_{s} / \hbar\right)\left|v_{k}^{\prime}\right\rangle\left\langle v_{k}^{\prime} \mid v_{j}\right\rangle=e^{i \phi_{j}}|j\rangle .
$$

Clearly, if

$$
\left\langle v_{k}^{\prime} \mid v_{j}\right\rangle=\exp \left(i \epsilon_{k}^{\prime} t_{s} / \hbar\right) e^{i \phi_{j}}\left\langle v_{k}^{\prime} \mid j\right\rangle
$$

then one would guarantee that

$$
U\left|v_{j}\right\rangle=e^{i \phi_{j}}\left(\sum_{k=0}^{N}\left|v_{k}^{\prime}\right\rangle\left\langle v_{k}^{\prime}\right|\right)|j\rangle=e^{i \phi_{j}}|j\rangle
$$

where the term in parentheses is unity because the eigenbasis of $H$ is complete.

It would be preferable if the system would evolve back to the original eigenbasis after an additional time $t_{s}$, at least up to an overall phase. This is equivalent to requiring that

$$
U^{2}=\sum_{k=0}^{N} \exp \left(-i 2 \epsilon_{k}^{\prime} t_{s} / \hbar\right)\left|v_{k}^{\prime}\right\rangle\left\langle v_{k}^{\prime}\right|=I e^{i \theta},
$$

where $I$ is the identity matrix of dimension $N+1$ and $\theta$ is some phase. This condition is most generally obtained when $2 \epsilon_{k}^{\prime} t_{s} / \hbar=2 \pi n-\theta$ for any $k, n$. Suppose that we choose $n=k^{m}$ with $m$ some arbitrary integer $(m=1$ implies the spectrum is linear, $m=2$ gives a quadratic spectrum, etc.). Then $\epsilon_{k}^{\prime}=\epsilon_{0}^{\prime}+c k^{m}$, with $\epsilon_{0}^{\prime}=-\hbar \theta / 2 t_{s}$ and $c=\hbar \pi / t_{s}$. Eq. (22) can now be written

$$
\begin{aligned}
U\left|v_{j}\right\rangle & =e^{-i \epsilon_{0}^{\prime} t_{s} / \hbar} \sum_{k=0}^{N} e^{-i c k^{m} t_{s} / \hbar}\left|v_{k}^{\prime}\right\rangle\left\langle v_{k}^{\prime} \mid v_{j}\right\rangle \\
& =e^{i \theta / 2} \sum_{k=0}^{N} e^{-i \pi k^{m}}\left|v_{k}^{\prime}\right\rangle\left\langle v_{k}^{\prime} \mid v_{j}\right\rangle \\
& =e^{i \theta / 2} \sum_{k=0}^{N}(-1)^{k}\left|v_{k}^{\prime}\right\rangle\left\langle v_{k}^{\prime} \mid v_{j}\right\rangle=e^{i \phi_{j}}|j\rangle .
\end{aligned}
$$


Thus, in order to perform a spectral transform that is invertible by repetition, one requires

$$
\left\langle v_{k}^{\prime} \mid v_{j}\right\rangle=(-1)^{k} e^{i\left(\phi_{j}-\theta / 2\right)}\left\langle v_{k}^{\prime} \mid j\right\rangle \quad \forall k, j \in\{0, N\}
$$

In short, the overlap of the eigenvectors of the full Hamiltonian $H$ with those of the unperturbed Hamiltonian $H_{0}$ must be trivially related to their overlap with the site basis vectors. Furthermore, the perturbation to $H_{0}$ must be local (i.e. be strictly an externally applied spatially dependent potential), and the spectrum of the full Hamiltonian must be an integer power in the quantum number.

Consider the homogeneous optical lattice with $J_{i, j}=J$ on $N$ sites. Hard-wall boundary conditions force all functions to zero at the sites labeled by $i=-1$ and $i=N+1$. The unperturbed eigenvalues in ascending order are $\epsilon_{k}=-2 J \cos [\pi(k+1) /(N+2)]$ with $k \in\{0, N\}$, and the eigenvectors are similar to Chebyshev polynomials, $\left|v_{k}\right\rangle=\sqrt{2 /(N+2)} \sin [\pi(k+1)(j+1) /(N+2)]$, where $k$ is the eigenvalue index and $j \in\{0, N\}$ corresponds to the lattice site. It is straightforward to verify that choosing $V_{k}=\epsilon_{k}$, i.e. where the perturbation corresponds to the eigenvalues of the original unperturbed Hamiltonian in ascending order, indeed yields perturbed eigenvectors $\left|v_{k}^{\prime}\right\rangle$ that satisfy the desired overlap relation $\left\langle v_{k}^{\prime} \mid v_{j}\right\rangle=(-1)^{k+j}\left\langle v_{k}^{\prime} \mid j\right\rangle$. Unfortunately, the spectrum of the perturbed Hamiltonian is not of the desired special form $\epsilon_{k}^{\prime}=\epsilon_{0}^{\prime}+c k^{m}$. Conversely, if a perturbation is chosen to enforce the eigenvalue criterion, then the overlap criterion cannot be satisfied. The unfortunate conclusion is that no perturbation to a uniform optical lattice can be chosen in order to effect a spectral transform. The remainder of this work is devoted to showing that both of these conditions can be nevertheless be simultaneously satisfied with atoms in specially prepared optical lattices.

\section{KRAWTCHOUK LATTICE}

Consider the site-dependent tunneling amplitudes

$$
J_{i, i+1} \equiv J \sqrt{(i+1)(N-i)}
$$

where $i \in\{0, N-1\}$ and $J$ is a bare tunneling coefficient with units of energy. The associated weighted lattice Hamiltonian has been recently investigated in the context of quantum walks [41, 42, 43, 44], the quantum mechanical generalization of the classical random walk [45, 46]. In particular, with these coefficients in the governing Hamiltonian, a quantum state initialized in the left-most lattice site will evolve with perfect probability to the rightmost lattice site after a fixed amount of time. For this reason, this Hamiltonian and its generalizations have been denoted a perfect quantum wires [47]. Propagating further for the same amount of time returns the state to the first site. Likewise, states at any site will be transferred to sites symmetrically located around the lattice center, so time-evolution effects a perfect reflection of any state about the midpoint. Much of the interest in this and related systems stems from their potential uses for quantum computation and communication, because of their ability to perfectly distribute quantum states and entanglement [48, 49].

In the present context the Hamiltonian (10) with tunneling coefficients (28) is useful because it possesses both a linear spectrum and exhibits periodic behavior. Indeed, the eigenfunctions turn out to be Krawtchouk functions, which are the discrete-space analogs of the Hermite polynomials. Furthermore, it will be shown below that perturbing the quantum wire by a linear potential will yield a full Hamiltonian whose states satisfy the criterion (27), thereby yielding a full spectral transformation between energy and coordinate space.

\section{A. Unperturbed Hamiltonian}

The spectrum of the unperturbed Hamiltonian (10) is obtained by solving $H_{0}\left|v_{k}\right\rangle=\epsilon_{k}\left|v_{k}\right\rangle$ Expanding the eigenfunctions in the site basis $\left|v_{k}\right\rangle \equiv \sum_{j} a_{j}^{(k)}|j\rangle$ with $a_{j}^{(k)}$ some unknown expansion coefficients, one can write the eigenvalue equation as

$$
\begin{gathered}
\sum_{i, j} a_{j}^{(k)}\left(J_{i+1, i}|i+1\rangle\langle i \mid j\rangle+J_{i-1, i}|i-1\rangle\langle i \mid j\rangle\right. \\
\left.-\epsilon_{k}|j\rangle\right)=0
\end{gathered}
$$

which can be conveniently rewritten as the three-term recurrence relation

$$
J_{j, j-1} a_{j-1}^{(k)}+J_{j, j+1} a_{j+1}^{(k)}-\epsilon_{k} a_{j}^{(k)}=0 .
$$

In terms of the quantum wire tunneling amplitudes (28) this becomes

$$
\begin{aligned}
& \sqrt{j(N-j+1)} a_{j-1}^{(k)}+\sqrt{(j+1)(N-j)} a_{j+1}^{(k)} \\
& -\tilde{\epsilon}_{k} a_{j}^{(k)}=0
\end{aligned}
$$

where the eigenvalues $\tilde{\epsilon}_{j} \equiv \epsilon_{j} / J$ are now dimensionless.

The recurrence relation (31) defines the normalized Krawtchouk polynomials $\psi_{j}^{(p)}(z)$ [27, 28]

$$
\begin{aligned}
& \sqrt{j(N-j+1)} \psi_{j-1}^{(p)}(k)+\sqrt{(j+1)(N-j)} \psi_{j+1}^{(p)}(k) \\
& +\frac{N p+(1-2 p) j-k}{\sqrt{p(1-p)}} \psi_{j}^{(p)}(k)=0
\end{aligned}
$$

where the Krawtchouk polynomials can be expressed in terms of either hypergeometric or Jacobi functions:

$$
\begin{aligned}
& \psi_{j}^{(p)}(k)=\left(\frac{p}{1-p}\right)^{\frac{k+j}{2}}(-1)^{j}(1-p)^{N / 2} \sqrt{\frac{j !(N-j) !}{k !(N-k) !}} \\
& \times\left(\begin{array}{c}
N \\
j
\end{array}\right){ }_{2} F_{1}\left(-j,-k ;-N ; \frac{1}{p}\right),
\end{aligned}
$$




$$
\begin{gathered}
=\left(\frac{p}{1-p}\right)^{\frac{k+j}{2}}(-1)^{j}(1-p)^{N / 2} \sqrt{\frac{j !(N-j) !}{k !(N-k) !}} \\
\times P_{k}^{(N-j-k,-N-1)}\left(\frac{2}{p}-1\right),
\end{gathered}
$$

with $p \in \mathbb{R}$ a free parameter. Comparison of Eqs. (31) and (32) immediately requires $p=1 / 2$ to eliminate the $j$ (position) dependence of the last coefficient. The spectrum of the unperturbed Hamiltonian is therefore linear, $\tilde{\epsilon}_{k}=-N+2 k$. The eigenvectors are

$$
\left|v_{k}(j)\right\rangle=\frac{(-1)^{j}}{2^{N / 2}} \sqrt{\frac{j !(N-j) !}{k !(N-k) !}} P_{k}^{(N-j-k,-N-1)}(3),
$$

where the position-dependence is now included explicitly.

In the limit of large $N$ and small $k$, the Krawtchouk polynomials reduce to Hermite polynomials [27, 50], reflecting the fact that the Hamiltonian for the quantum wire is the discretized version of the harmonic oscillator. This is easy to verify directly from the recurrence relation (31) in the limit $N \rightarrow \infty$ and $j \ll N$ :

$$
\sqrt{j N} a_{j-1}^{(k)}+\sqrt{(j+1) N} a_{j+1}^{(k)}+(N-2 k) a_{j}^{(k)}=0,
$$

which is equivalent to the recurrence relation for the normalized Hermite polynomials $\tilde{H}_{j}(x)$ [51]

$$
\sqrt{j} \tilde{H}_{j-1}+\sqrt{j+1} \tilde{H}_{j+1}-x \sqrt{2} \tilde{H}_{j}=0 .
$$

Evidently, $x=\sqrt{2} k$ neglecting the overall constant term. The effective trapping (oscillation) frequency is therefore $\omega=2 J / \hbar$.

\section{B. Full Hamiltonian}

The $j$-dependence of the third coefficient appearing in the recurrence relation (32) indicates that adding a linear potential $V_{j} \propto j$ in (19) to the unperturbed Hamiltonian would still yield eigenstates that are Krawtchouk functions. In this case the potential would need to be of the form

$$
\tilde{V}_{j}=\frac{1-2 p}{\sqrt{p(1-p)}} j
$$

yielding the softened linear spectrum

$$
\epsilon_{k}^{\prime}=\frac{1}{\sqrt{p(1-p)}}(-N p+k)
$$

and the full expression (33) for the eigenvectors $\left|v_{k}^{\prime}(j)\right\rangle=$ $\psi_{j}^{(p)}(k)$.

It remains to be shown that there is a choice of $p$ for the full Hamiltonian that can satisfy the main condition (27). In fact, as will be proven below, there is indeed a choice of $p$ that ensures

$$
\left\langle v_{k}^{\prime} \mid v_{m}\right\rangle=(-1)^{k+m}\left\langle v_{k}^{\prime} \mid m\right\rangle,
$$

which effects the spectral transformation $U\left|v_{m}\right\rangle=$ $(-1)^{m}|m\rangle$ for all $m$, and another choice that performs the mirror-symmetric version $U\left|v_{m}\right\rangle=(-1)^{m}|N-m\rangle$. In terms of the Krawtchouk functions, condition (39) can be written as

$$
\begin{aligned}
& \sum_{j=0}^{N}\left(\begin{array}{c}
N \\
j
\end{array}\right)^{-1} \frac{(-1)^{k+m}}{2^{N / 2}}\left(\begin{array}{c}
N \\
m
\end{array}\right) P_{j}^{(N-j-m,-N-1)}(3) \\
& \times\left(\frac{p}{1-p}\right)^{j / 2}\left(\begin{array}{c}
N \\
k
\end{array}\right) P_{j}^{(N-j-k,-N-1)}\left(\frac{2}{p}-1\right) \\
& =\left(\frac{p}{1-p}\right)^{m / 2}\left(\begin{array}{c}
N \\
k
\end{array}\right) P_{m}^{(N-m-k,-N-1)}\left(\frac{2}{p}-1\right) .
\end{aligned}
$$

Eq. (40) can be thought of as an $(N+1)$-dimensional matrix equation $K_{1} K_{2}=K_{3}$, with the sum over $j$ on the left-hand side effecting the product of the two Krawtchouk matrices 52, 53

$$
\begin{array}{r}
K_{1}^{(N)}=\frac{(-1)^{k}}{2^{N / 2}}\left(\begin{array}{c}
N \\
m
\end{array}\right) P_{j}^{(N-m-j,-N-1)}(3), \\
K_{2}^{(N)}=(-1)^{m}\left(\frac{p}{1-p}\right)^{j / 2}\left(\begin{array}{c}
N \\
k
\end{array}\right) \\
\times P_{j}^{(N-j-k,-N-1)}\left(\frac{2}{p}-1\right),
\end{array}
$$

which are clearly symmetric under the interchange of row and column indices $j \leftrightarrow m$ or $j \leftrightarrow k$ respectively, and the right hand side given by the symmetric Krawtchouk matrix

$$
K_{3}^{(N)}=\left(\frac{p}{1-p}\right)^{m / 2}\left(\begin{array}{c}
N \\
k
\end{array}\right) P_{m}^{(N-m-k,-N-1)}\left(\frac{2}{p}-1\right) .
$$

The factor $\left(\begin{array}{c}N \\ j\end{array}\right)$ in the sum (40) serves as a "metric tensor,' as described in greater detail below. The Krawtchouk matrix elements are found by fixing $N$ and treating the two variables in the superscript of the Jacobi polynomials as the row and column indices, respectively. Thus, the columns of the symmetric Krawtchouk matrices $K_{1}^{(N)}$ and $K_{2}^{(N)}$ correspond to the eigenvalue-ordered eigenvectors of the unperturbed and perturbed Hamiltonians $H_{0}$ and $H_{1}$ on $N+1$ sites, respectively.

As has been recently discussed in Ref. 53, Krawtchouk matrices correspond to the 'Hamming contraction' of tensor products of identical matrices. The hierarchy of Krawtchouk matrices is mathematically expressed as $K^{(N)}=r\left\{\left(K^{(1)}\right)^{\otimes N}\right\}$, where $K^{(N)}$ is the $N$ th order Krawtchouk matrix of dimension $N+1$, and $r$ is an operator effecting the Hamming contraction. The contraction is effected by labeling the rows/columns of the contracted matrix by the Hamming weight of the row/column index (written in binary) for the original matrix, and summing 
all matrix elements whose row (column) indices share the same Hamming weight. For example, the first-order Krawtchouk matrix $K_{1}^{(1)}$ is the Hadamard operator $\bar{H}$

$$
K_{1}^{(1)}=\frac{1}{\sqrt{2}}\left(\begin{array}{cc}
1 & 1 \\
1 & -1
\end{array}\right)=\bar{H},
$$

and the second-order Krawtchouk matrix $K_{1}^{(2)}$ is the contraction of the tensor product of two Hadamard operators:

$$
\begin{aligned}
K_{1}^{(2)} & =r(\bar{H} \otimes \bar{H})=r\left[\frac{1}{2}\left(\begin{array}{cccc}
1 & 1 & 1 & 1 \\
1 & -1 & 1 & -1 \\
1 & 1 & -1 & -1 \\
1 & -1 & -1 & 1
\end{array}\right)\right] \\
& =\frac{1}{2}\left(\begin{array}{ccc}
1 & 2 & 1 \\
2 & 0 & -2 \\
1 & -2 & 1
\end{array}\right) .
\end{aligned}
$$

Equation (40) can therefore be interpreted as the product of uncontracted tensor products of first-order Krawtchouk matrices,

$$
\left(K_{1}^{(1)}\right)^{\otimes N}\left(K_{2}^{(1)}\right)^{\otimes N}=\left(K_{3}^{(1)}\right)^{\otimes N},
$$

which can be alternatively expressed as

$$
\left(K_{1}^{(1)} K_{2}^{(2)}\right)^{\otimes N}=\left(K_{3}^{(1)}\right)^{\otimes N}
$$

The 'weight factor' $\left(\begin{array}{c}N \\ j\end{array}\right)$ appearing in the sum of Eq. (40) can now clearly be understood as accounting for the multiplicities for each Hamming weight on the integers between 0 and $2^{N}$, and therefore serves as a 'metric' for matrix-matrix multiplications in contracted space. Thus, to prove the equality (40) for arbitrary $N$ one need only find the value of $p$ satisfying the $N=1$ case:

$$
\begin{gathered}
\frac{1}{\sqrt{2}}\left(\begin{array}{cc}
1 & 1 \\
1 & -1
\end{array}\right)\left(\begin{array}{cc}
1 & 1 \\
\sqrt{\frac{p}{1-p}} & -\sqrt{\frac{1-p}{p}}
\end{array}\right) \\
=\frac{1}{\sqrt{2}}\left(\begin{array}{cc}
1 & -1 \\
\sqrt{\frac{p}{1-p}} & \sqrt{\frac{1-p}{p}}
\end{array}\right),
\end{gathered}
$$

which yields the unique solution

$$
p=\frac{1}{4}(2-\sqrt{2}) .
$$

It is easy to verify that this solution indeed satisfies Eq. (40) for arbitrary choices of $N$. Inserting this result into Eqs. (37) and (38) gives the linear potential

$$
\tilde{V}_{j}=2 j
$$

and the resulting linear spectrum for the perturbed Hamiltonian

$$
\epsilon_{k}^{\prime}=\sqrt{2}(-N+2 k) .
$$

Because $c=2 \sqrt{2}$ in Eq. (26), the time to effect the spectral transform is

$$
t_{s}=\frac{\pi}{2 \sqrt{2}}\left(\frac{\hbar}{J}\right) .
$$

Eqs. (28), (50) and (52) constitute the main results of this work.

To perform the mirror-symmetric spectral transform $U\left|v_{m}\right\rangle=|N-m\rangle$ one needs to obtain the value of $p$ that ensures

$$
\left\langle v_{k}^{\prime} \mid v_{m}\right\rangle=(-1)^{k+m}\left\langle v_{k}^{\prime} \mid N-m\right\rangle,
$$

In this case, one obtains

$$
\begin{aligned}
K_{3}^{(N)} & =(-1)^{k+m}\left(\frac{p}{1-p}\right)^{m / 2}\left(\begin{array}{c}
N \\
k
\end{array}\right) \\
& \times P_{N-m}^{(m-k,-N-1)}\left(\frac{2}{p}-1\right) .
\end{aligned}
$$

Following the same procedure as discussed above, it is straightforward to show that to satisfy condition (53) one requires $p=\frac{1}{4}(2+\sqrt{2})$. This yields the same prefactor as the solution above, namely $1 / \sqrt{p(1-p)}=2 \sqrt{2}$. The spectrum is therefore the same, but the applied potential now has the opposite sign, $\tilde{V}_{j}=-2 j$. Thus, to perform an inverted spectral transform where the ground state is transformed to the rightmost site rather than the leftmost, one simply requires the same linear potential but with a negative slope.

\section{Discussion of Main Results}

One might be surprised that neither the applied linear potential (50) nor the required time (52) depend explicitly on the number of lattice sites. Clearly, as $N$ grows, the initial wavepacket must traverse an increasing number of lattice sites during the implementation of the spectral transform. It is important to recall, however, that the actual tunneling amplitudes (28) do indeed increase with $N$. That said, it is not obvious that their values are just what are required to ensure that $t_{s}$ remains constant. Consider the time it takes on average to tunnel between adjacent lattice sites, $\overline{t_{i, i+1}} \approx h / J_{i, i+1}$. The total time required for a wavepacket to propagate from one end of the lattice to the other is then approximately

$$
\bar{T} \approx \frac{\hbar}{J} \sum_{i=0}^{N} \frac{1}{\sqrt{(i+1)(N-i)}} .
$$

In the large- $N$ limit, one obtains the asymptotic result $\lim _{N \rightarrow \infty} \bar{T}=(\hbar / J) \pi=h / 2 J$. This is exactly the period for one oscillation of the original Krawtchouk oscillator: the eigenvalues $\tilde{\epsilon}_{k}=2 k$ are equivalent to the frequency $2 J / \hbar=2 \pi / T$, which yields $T=h / 2 J$.

The fact that the Krawtchouk matrices represent contractions of tensor products of identical matrices has 
in fact been anticipated in the literature, though in a completely different context. The quantum wire tunneling amplitudes (28) are obtained by 'collapsing' the hypercube graph to a weighted linear graph, by rendering indistinguishable vertex labels with the same Hamming weight [41]. The hypercube is constructed by tensor (Cartesian) products of two-site linear graphs. The eigenvectors are therefore tensor products of the two solutions for the two-site graph. These solutions are represented by the Hadamard matrix $\bar{H}$. The Hamiltonian and its eigenvectors for the quantum wires (the collapsed hypercubes) are therefore contractions of the associated hypercube quantities.

It should be pointed out that the relationship between Krawtchouk functions and spectral transforms has been discussed previously in the literature, primarily in the context of optical and digital filtering [54, 55]. These ideas were extended to quantum mechanical systems, where the Krawtchouk transform can be considered as a fractional Fourier transform [50]. That said, the idea presented in this paper of performing the spectral tranform by time-evolution under the influence of a local operator is entirely new.

It is also important to mention that the discrete nature of the Hamiltonian is a crucial ingredient that enables the spectral transform to be performed simply by turning on a linear potential. Suppose we attempted the same approach in the continuum limit $N \rightarrow \infty$ in which the Krawtchouk functions reduce to Hermite polynomials. In this limit, the inhomogeneous tight-binding Hamiltonian is replaced by a harmonic oscillator Hamiltonian with a potential $V(z)=\frac{1}{2} m \omega^{2} z^{2}$ with $\omega=2 J / \hbar$ as discussed at the end of Sec. IVA Adding a linear potential $V(z)=$ $2 J[z /(\lambda / 2)]=4 J z / \lambda$, the continuum limit of the discrete linear potential $\tilde{V}_{j}=2 j$ required to effect the spectral transform, the total potential becomes

$$
V(z)=\frac{1}{2} m \omega^{2}\left(z+\frac{4 J}{m \omega^{2} \lambda}\right)^{2}-\frac{8 J^{2}}{m \omega^{2} \lambda^{2}} .
$$

The linear potential simply shifts the origin of the effective oscillator potential to the left, so the eigenstates of the unperturbed Hamiltonian are instantaneously placed at the classical turning points of the shifted potential. The subsequent evolution is simple harmonic motion, with all wavepackets initially propagating to the left. Under no circumstances will certain eigenstates propagate to the right!

One more note should be made before discussing the feasibility of performing this spectral transform in actual optical lattices in the next section. The potential that must to be applied $(V(j)=2 J j)$ in order to effect the spectral transform corresponds exactly to the eigenvalues of the original (unperturbed) Hamiltonian, listed along the diagonal in increasing order. Curiously, this was exactly the same criterion found to satisfy the condition (27) for the uniform lattice, discussed at the end of Sec. III though in that case the full spectral transform could not be implemented because the resulting eigenvalue spectrum was not an integer power-law. This observation could provide a tantalizing clue to generalizing the spectral transform by Hamiltonian evolution for other discrete systems.

\section{APPROXIMATIONS AND INTERACTIONS}

The implementation of a spectral transform by Hamiltonian evolution described in the previous section assumed that there is only one particle in the entire lattice. This is of course not generally the case in real experiments, where there are many atoms and collisions between pairs of particles is not negligible. It is therefore important to investigate the efficacy of the approach including the effects of particle interactions. This is accomplished by including the interaction term proportional to $U$ in the Bose-Hubbard Hamiltonian (3), which has been so far neglected.

Likewise, it has been assumed that the site-dependent tunneling coefficients $J_{i, i+1}$ in (28) could be implemented in actual experiments. In practice, the tunneling amplitudes can be made to vary continuously over the length of the lattice, as discussed in Sec. III, but this variation is unlikely to yield exactly the correct values of the $J_{i, i+1}$. This section considers separately the robustness of the spectral transform when the exact conditions discussed in Sec. III are no longer satisfied.

\section{A. Quadratic Optical Lattice}

The exact tunneling coefficients (28) required for the spectral transform would not be straightforward to implement experimentally. As discussed in Sec.II] a natural variation of the lattice depth arises from the laser beam waists $z_{0}, V(z)=V_{0} \exp \left(-z^{2} / z_{0}^{2}\right)$. With the substitution $z=(\lambda / 2) i$, the axial variation for the discrete case is $V_{i}=V_{0} \exp \left[-\left(\lambda / 2 z_{0}\right)^{2} i^{2}\right]$. Inserting this into the tightbinding expression for the hopping coefficients (5) and assuming that $z_{0} \gg \lambda$ one obtains

$$
\frac{J_{i, i+1}}{E_{R}} \approx J_{0}\left[1+\left(\frac{\lambda}{2 z_{0}}\right)^{2}\left(\sqrt{\bar{V}_{0}}-\frac{3}{4}\right) i^{2}\right],
$$

where $J_{0}=\sqrt{\frac{16}{\pi}} \bar{V}_{0}^{3 / 4} e^{-2 \sqrt{\bar{V}_{0}}}$ and terms of order $\left(\lambda / 2 z_{0}\right)^{4}$ have been neglected. Assuming $z_{0}=100 \mu \mathrm{m}$, $\lambda=0.8 \mu \mathrm{m}$, and $\bar{V}_{0}=30$, this quadratic approximation gives $J_{i, i+1} / E_{R} \approx J_{0}\left(1+7.5 \times 10^{-5} i^{2}\right)$. Can such a quadratic variation of the tunneling coefficients reproduce the desired values (28)?

In the vicinity of the lattice center $i=(N-1) / 2+j$ with $j$ a small integer $j \ll N$, the tunneling coefficients (28) are indeed approximately quadratic:

$$
J_{j, j+1}=J \sqrt{\left(\frac{N+1}{2}\right)^{2}-j^{2}}
$$




$$
\approx \frac{N+1}{2} J\left(1-\frac{2 j^{2}}{(N+1)^{2}}\right),
$$

neglecting terms of order $\mathcal{O}\left(j^{4}\right)$. While this is an excellent approximation near the lattice center, the tunneling coefficients decrease more quickly near the lattice edges; clearly, if $j=(N-1) / 2$ in the expression above one obtains $J_{(N-1) / 2,(N+1) / 2} \approx N / 4$, which is much larger than the exact tunneling coefficient $J_{(N-1) / 2,(N+1) / 2}=\sqrt{N}$. An alternative approximation that reproduces the desired values at both $j=0$ and $j= \pm(N-1) / 2$ would be

$$
J_{j, j+1}=\frac{N+1}{2} J\left(1-\frac{4 j^{2}}{(N+1)(\sqrt{N}+1)^{2}}\right) .
$$

Comparison of Eqs. (57) and (60) reveals a problem, however: the coefficients of the site-dependent term have opposite signs. This is because the actual tunneling coefficients for a focused laser beam (57) are smallest when the lattice depth (laser intensity) is greatest. In contrast, the desired coefficients (60) are supposed to be largest at the center, so that the lattice depth must be shallowest at this point.

\section{Axially displacing lattice beams}

In order to prepare an optical lattice with the desired properties, one could axially displace the lattice beams relative to the each other, so that the intensity maxima are located a distance $d \gg z_{0}$ apart. The lattice potential would then be $\bar{V}(z)=\frac{1}{2} \bar{V}_{0}\left(e^{-z^{2} / z_{0}^{2}}+e^{-(z-d)^{2} / z_{0}^{2}}\right)$. Written in terms of the new variable $x=z-d / 2$ centered midway between the two maxima, the potential becomes

$$
\bar{V}(x)=\bar{V}_{d / 2} e^{-x^{2} / z_{0}^{2}} \cosh \left(\frac{x d}{z_{0}^{2}}\right),
$$

where $\bar{V}_{d / 2}=\bar{V}_{0} e^{-d^{2} / 4 z_{0}^{2}}$ is the zeroth-order potential depth at the lattice center equidistant from the centers of the two beam maxima. Expanding about $x=0$ gives an approximate quadratic envelope

$$
\bar{V}(x) \approx \bar{V}_{d / 2}+\bar{V}_{d / 2} \frac{x^{2}}{2 z_{0}^{2}}\left(\frac{d^{2}}{z_{0}^{2}}-2\right)
$$

corresponding to tunneling coefficients

$$
J(x) \approx J_{d / 2}\left[1-\frac{x^{2}}{2 z_{0}^{2}}\left(\frac{d^{2}}{z_{0}^{2}}-\frac{1}{2}\right)\left(\sqrt{\bar{V}_{d / 2}}-\frac{3}{4}\right)\right],
$$

where $J_{d / 2}=\sqrt{\frac{16}{\pi}} \bar{V}_{d / 2}^{3 / 4} e^{-2 \sqrt{\bar{V}_{d / 2}}}$. Comparison of (60) and (63) immediately gives $J=2 J_{d / 2} /(N+1)$ and

$$
\frac{\lambda^{2}}{z_{0}^{2}}\left(\frac{d^{2}}{z_{0}^{2}}-\frac{1}{2}\right)\left(\sqrt{\bar{V}_{d / 2}}-\frac{3}{4}\right)=\frac{32}{(N+1)(\sqrt{N}+1)^{2}},
$$

where the substitution $x=(\lambda / 2) j$ has been made. Of course, to enforce the hard-wall boundary condition, end caps [34, 35] must also be applied transversally at the optical lattice amplitude maxima located at $z=0$ and $z=d$.

According to Eq. (8), the spatial variation of the potential depth (62) would yield a site-dependent energy shift that is not included in the assumed hopping Hamiltonian (10). Using the harmonic oscillator approximation to the Wannier functions yields $\varepsilon(x) / E_{R} \approx \sqrt{\bar{V}_{d / 2}} x^{2} d^{2} / 4 z_{0}^{4}$ ignoring the constant offset energy $\sqrt{\bar{V}_{d / 2}}$. It is therefore necessary to manually apply an inverted parabolic potential, centered at the depth minimum, that can serve to cancel this effective quadratic potential energy contribution.

Let's consider what these results imply for actual experiments, assuming ${ }^{87} \mathrm{Rb}$ atoms confined in a reddetuned optical lattice with wavelength $\lambda=0.8 \mu \mathrm{m}$. With $V_{0}=50 E_{R}$ and $z_{0}=100 \mu \mathrm{m}$, one could obtain a lattice with $N=201$ sites choosing $d \approx 255 \mu \mathrm{m}$. The potential depth at the midpoint between beam maxima is $V_{d / 2} \approx 10 E_{R}$, deeply within the tight-binding limit. The coupling constant is then calculated to be $J \approx 2.3 \times 10^{-4} E_{R}$, though the actual coupling constants vary from $0.01 E_{R}$ at the edge to $0.02 E_{R}$ at the midpoint between laser maxima. An external potential must be applied at all times, $\bar{V}_{\mathrm{e}}^{0}(x) \approx-5.1\left(x / z_{0}\right)^{2}$ so that the magnitude of the external potential reaches almost one recoil energy at the lattice edges. Applying the additional linear potential $V_{\mathrm{e}}^{1}(x)=4 J(x / \lambda)$ then allows for the implementation of the spectral transform in a time $t_{s}=\hbar \pi / 2 \sqrt{2} J \approx 210 \mathrm{~ms}$.

Likewise, for a lattice with $N=301$ sites and $V_{0}=$ $20 E_{R}$, one requires $d \approx 223 \mu \mathrm{m}$ which yields $V_{d / 2} \approx$ $5.8 E_{R}$. The external quadratic potential that needs to be applied is $\bar{V}_{\mathrm{e}}^{0}(x) \approx-3\left(x / z_{0}\right)^{2}$. With $J=4.6 \times 10^{-4} E_{R}$ (the actual coupling constants vary between $0.036 E_{R}$ and $\left.0.069 E_{R}\right)$, the time required is faster than for the example above, $t_{s}=107 \mathrm{~ms}$. It might at first glance appear paradoxical that the $N=301$ case should be faster than the $N=201$ case, but recall that the total time $t_{s}$ is set by the parameter $J$, which for the latter case is twice as large as the value for the former case.

In order to verify that the quadratic optical lattice is indeed able to perform the spectral transform, an explicit numerical simulation of the time evolution was performed. The governing tight-binding Hamiltonian is the unperturbed $H_{0}$ defined in Eq. (10), but with the approximate tunneling coefficients $J_{i, j}=J(j)=J(x)$ given in Eq. (63); its eigenvalues and eigenvalues and eigenvectors are $\epsilon_{k}$ and $\left|v_{k}\right\rangle$, respectively. For simplicity, the initial state was chosen to be a Boltzmann distribution

$$
\rho=\sum_{k} \exp \left(-\epsilon_{k} / k_{B} T\right)\left|v_{k}\right\rangle\left\langle v_{k}\right|,
$$

where $\rho$ is the thermal density matrix, $T$ is the temperature in Kelvin, and $k_{B} \approx 1.38 \times 10^{-23} \mathrm{~J} / \mathrm{K}$ is Boltz- 

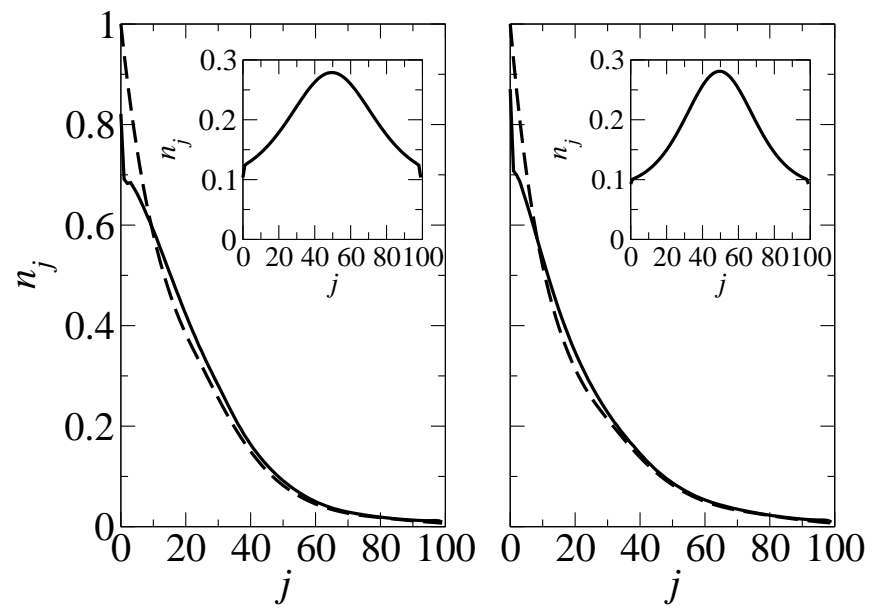

FIG. 1: Numerical results for the spectral transform. The parameters for the simulation are $k_{B} T=40 E_{R}, z_{0}=100 \mu \mathrm{m}$, $V_{0}=50 E_{R}$, and $N=100$. The left and right graphs correspond to $d=200 \mu \mathrm{m}$ and $300 \mu \mathrm{m}$, respectively. The results for the perfect tunneling coefficients (28) are given by dashed lines, while those based on tunneling coefficients (5) derived from the actual lattice potential (61) are given by solid lines. The insets depict the density profile of the initial thermal distribution for the actual lattice.

mann's constant. The time-evolution of the density matrix is obtained in principle by numerically integrating the equation of motion $i \hbar \partial \rho / \partial t=\left[H_{0}+4 J x / \lambda, \rho\right]$ where [,] denotes the commutator and the correct linear potential has been explicitly added. Equivalently, because the particles are assumed to have no interactions, one may simply allow each eigenvector of the unperturbed Hamiltonian to evolve separately under the influence of the Schrödinger equation, and then add their contributions to obtain the time-dependent density matrix

$$
\rho(t)=\sum_{k} \exp \left(-\epsilon_{k} / k_{B} T\right)\left|v_{k}(t)\right\rangle\left\langle v_{k}(t)\right|
$$

The spatially dependent particle density is then readily obtained using the relation $n(j, t)=\langle j|\rho(t)| j\rangle$ or $n(x, t)=\langle x|\rho(t)| x\rangle$.

The numerical results for the representative set of parameters $k_{B} T=40 E_{R}, z_{0}=100 \mu \mathrm{m}, V_{0}=50 E_{R}$, and $N=100$ are shown in Fig. 1 for the choices $d=200 \mu \mathrm{m}$ and $300 \mu \mathrm{m}$. Two cases were considered. In the first, the tunneling coefficients were chosen to be the exact ideal tunneling coefficients (28). As expected, the initial thermal distribution over energies is exactly reproduced after a time $t=\pi / 2 \sqrt{2} J$ as a spatial distribution over lattice sites $j$, shown as the dashed lines in the figure. In the second case, the tunneling coefficients were obtained for the actual lattice by inserting the spatially dependent optical lattice potential (61) into the general expression (5) appropriate in the tight-binding limit. The results are shown as solid lines in the figure. It is clear that for both values of $d$, the actual optical lattice does an ex- cellent job of reproducing the spectral transform. This indicates that a careful adjustment of the separation of the intensity maxima for the two optical lattice lasers (subject to $d \gg z_{0}$ ) is not necessary to ensure a successful implementation of the spectral transform. These general conclusions are also found to be independent of temperature.

It is also worthwhile to point out that the ability to perform the spectral transform directly in the optical lattice greatly simplifies both the measurement of temperature and the ability to cool the atoms. The initial particle density (shown as insets in Fig. 1) is very broad, with tails that are strongly truncated by the lattice edges. This would make a direct measurement of temperature, obtained by fitting the tails to an exponential function, quite unreliable. Furthermore, while one could attempt to cool the initial cloud directly by ejecting particles far from the lattice center, one would have little control over the actual energies that were removed from the system. In contrast, the particle density after the time propagation is almost an exact spatial map of the initial energy distribution. The exponential tail (at least for $k_{B} T<V_{0}$ ) is well-resolved, allowing an excellent determination of temperature. Furthermore, evaporative cooling on this state simply requires the removal of all atoms occupying sites with index $j \gtrsim 1$, followed by another spectral transform back to the energy basis.

\section{Spatial variation by interference}

While the approach described above of offsetting the focal distances of the two laser beams is able to reproduce the desired spectral transform, many experiments are performed under the assumption that the hopping amplitudes are approximately constant over the region of interest. It would be preferable to employ a homogeneous optical lattice (with hard-wall boundary conditions) for experiments, and then to only introduce the spatial inhomogeneity when temperature measurements or cooling is desired. Unfortunately, changing the focal length in the middle of an experiment is not feasible.

An alternative approach to modulate the optical lattice is through interference. Suppose that the homogeneous lattice is formed from two counter-propagating laser beams each with amplitude $A$ and frequency $\Omega$ in the standard lin- $-\perp$-lin configuration, i.e. where the two polarizations $\vec{\epsilon}$ perpendicular to one another. The electric field is then

$$
\begin{aligned}
\vec{E}(z) & =A \epsilon_{x} e^{i 2 \pi z / \lambda} e^{-i \Omega t}+A \epsilon_{y} e^{-i 2 \pi z / \lambda} e^{-i \Omega t} \\
& =A e^{i 2 \pi z / \lambda} e^{-i \Omega t}\left(\epsilon_{x}+\epsilon_{y} e^{-i 4 \pi z / \lambda}\right)
\end{aligned}
$$

The spatial variation of the polarization vector gives rise to a periodic atomic polarizability, which in turn yields the lattice potential $V(z) \propto \cos ^{2}(2 \pi z / \lambda)$. If one now introduces a second beam with electric field amplitude 
$B \ll A$ oriented in the $y z$-plane at a small angle $\theta$ with respect to the $z$-axis,

$$
\begin{aligned}
\vec{E}_{2}(z) & =B e^{-i \Omega t}\left\{\epsilon_{x} e^{i 2 \pi[z \cos (\theta)+y \sin (\theta)] / \lambda}\right. \\
& \left.+\epsilon_{y} e^{-i 2 \pi[z \cos (\theta)+y \sin (\theta)] / \lambda}\right\}
\end{aligned}
$$

then the total lattice potential becomes

$$
V(z)=V_{0}\left\{\cos \left(\frac{2 \pi z}{\lambda}\right)+\alpha \cos \left[\frac{2 \pi z}{\lambda} \cos (\theta)\right]\right\}^{2},
$$

where $\alpha \propto|B|^{2} /|A|^{2} \ll 1$ is the relative depth of the second lattice beam.

For small angle $\theta \ll 2 \pi$ so that $\cos (\theta) \approx 1-\theta^{2} / 2$, Eq. (69) can be rewritten as

$$
V(z) \approx V_{0}\left\{\cos \left(\frac{2 \pi z}{\lambda}\right)+\alpha \cos \left(\frac{2 \pi z}{\lambda}-\frac{2 \pi z}{\lambda_{b}}\right)\right\}^{2}
$$

where the 'beat wavelength' has been defined as $\lambda_{b} \equiv$ $2 \lambda / \theta^{2} \gg \lambda$, for reasons that will be clear shortly. The potential then becomes

$$
\begin{aligned}
V(z) \approx & V_{0}\left[1+\alpha \cos \left(\frac{2 \pi z}{\lambda_{b}}\right)\right]^{2} \cos ^{2}\left(\frac{2 \pi z}{\lambda}\right) \\
& +\alpha \sin \left(\frac{4 \pi z}{\lambda}\right) \sin \left(\frac{2 \pi z}{\lambda_{b}}\right) .
\end{aligned}
$$

If $\alpha$ is small then the last term in the above expression is small and can be neglected. The potential is therefore described by a spatially varying depth $V_{0}\left[1+\alpha \cos \left(2 \pi z / \lambda_{b}\right)\right]^{2}$ which is periodic at the beat wavelength. Unlike the case $\alpha=1$ often encountered in physics, however, the potential depth never vanishes: the maximum depth $V_{\max } \approx V_{0}(1+2 \alpha)$ corresponds to $z \approx 0$, while the minimum depth for $z \approx \lambda_{b} / 2=\lambda / \theta^{2}$ is $V_{\min } \approx V_{0}(1-2 \alpha)$.

Expanding about the minimum $z=x+\lambda_{b} / 2$ in the regime $x \ll \lambda_{b}$ one obtains

$$
\bar{V}(x) \approx \bar{V}_{0}\left[1-2 \alpha+\frac{4 \pi^{2} \alpha}{\lambda_{b}^{2}} x^{2}\right] \cos ^{2}\left(\frac{2 \pi x}{\lambda}\right),
$$

where as usual $\bar{V} \equiv V / E_{R}$. Thus, again the lattice depth is approximately a quadratic function of the lattice position. This leads to a quadratic site-dependent energy contribution (8):

$$
\bar{\varepsilon}(x) \approx \sqrt{\frac{\bar{V}_{0}}{1-2 \alpha}} \frac{2 \pi^{2} \alpha x^{2}}{\lambda_{b}^{2}}
$$

neglecting site-independent energies. Substituting this expression into Eq. (5) yields the site-dependent tunneling coefficients in vicinity of the depth minimum

$$
J(x) \approx J_{\lambda_{b} / 2}\left[1-4 \alpha \pi^{2} \frac{x^{2}}{\lambda_{b}^{2}}\left(\sqrt{V_{0}}-\frac{3}{4}\right)\right],
$$

where

$$
J_{\lambda_{b} / 2}=J_{0}\left[1+2 \alpha\left(\sqrt{V_{0}}-\frac{3}{4}\right)\right]
$$

is the tunneling amplitude at the lattice minimum in terms of the bare tunneling amplitude $J_{0}$.

Comparison with Eq. (60) gives $J=2 J_{\lambda_{b} / 2} /(N+1)$ and

$$
\frac{4}{(N+1)(\sqrt{N}+1)^{2}}=\alpha\left(\frac{\pi \lambda}{\lambda_{b}}\right)^{2}\left(\sqrt{V_{0}}-\frac{3}{4}\right) .
$$

It is again useful to insert numbers that would be relevant to experiments with ultracold atoms. With $\theta=\pi / 25$, the beat wavelength is $\lambda_{b}=126.6 \lambda$ or approximately 253 lattice spacings. One should therefore be able to effect a spectral transform with $N \lesssim 201$. Choosing $V_{0}=$ $10 E_{R}$ and $N=101$, condition (176) yields $\alpha \approx 0.216$, giving $J \approx 9 \times 10^{-4} E_{R}$ and therefore $t_{s} \approx 54 \mathrm{~ms}$. Note that an inverted parabolic potential $\bar{V}_{\mathrm{e}}^{0}(x) \approx-18\left(x / \lambda_{b}\right)^{2}$ must be applied at all times to cancel the site-dependent energy contribution (73). Likewise, choosing $N=201$ requires $\alpha \approx 0.058$ and $t_{s} \approx 170 \mathrm{~ms}$. For larger values of $N$, one would require smaller values of $\theta$ to ensure that $\lambda_{b} \gg N(\lambda / 2)$.

The main advantage of this approach is that temperature measurements and/or cooling can be effected at any stage of an experiment with ultracold atoms in optical lattices: one simply adds the second lattice beam and the appropriate quadratic and linear potentials. Of course, these must be added adiabatically in order to avoid heating the system in the process. A conservative estimate of the adiabaticity timescale would correspond to the time taken by a wavepacket to propagate fully across the lattice a few times, $t_{\text {adiab }} \approx 2 \hbar / J$ from Eq. (55). For the $N=101$ example above, one obtains $t_{\text {adiab }} \approx 100 \mathrm{~ms} \mathrm{so}$ that a ramp-up of the additional beam and the external quadratic potential over 200 to $300 \mathrm{~ms}$ would ensure that the system would be minimally perturbed.

\section{B. Effect of Interactions}

Until now the interaction term in the Bose-Hubbard Hamiltonian (3) has been completely neglected. In principle, the strength of particle interactions can be adjusted close to zero through the use of Feshbach resonances [56], but in general the gas will not be perfectly ideal. Unfortunately, a full numerical simulation of the spectral transform, as was discussed in the subsection above, is not straightforward in the presence of interactions. The interactions lead to mixing among the energy levels during the time propagation that are impossible to model in an average way without introducing additional assumptions.

Instead, the spectral transform was numerically simulated by exact propagation of the many particle Hamiltonian, but for a smaller number of lattice sites $N=10$. 


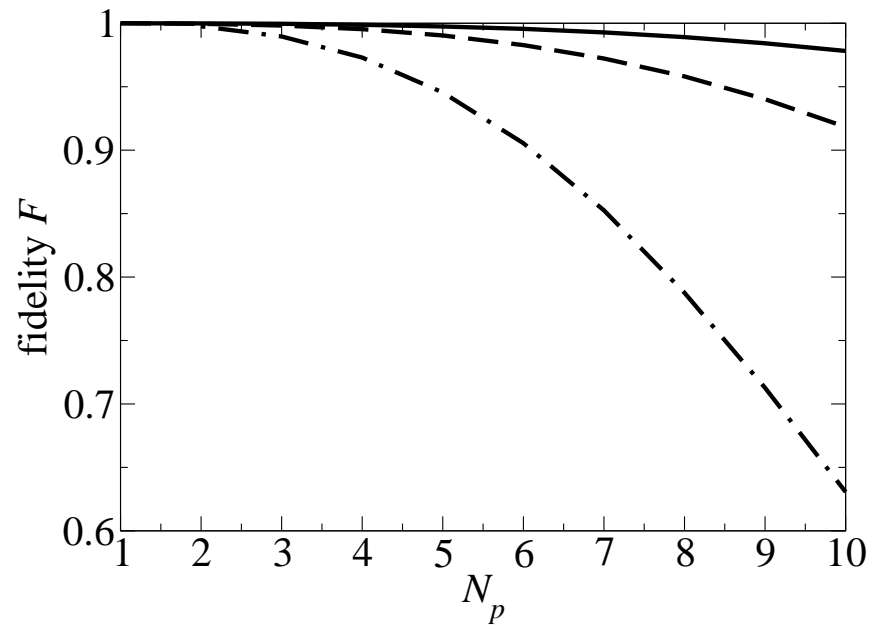

FIG. 2: Fidelity $F$ as a function of the number of particles $N_{p}$ obtained by exact numerical simulation of the spectral transform. With the initial state the ground state of the unperturbed interacting Hamiltonian, the fidelity is defined as the density in the first site at time $t_{s}=\pi / 2 \sqrt{2} J$. The solid, dashed, and dot-dashed lines correspond to $U=0.1 \mathrm{~J}, 0.2 \mathrm{~J}$, and $0.5 J$, respectively.

The tunneling coefficients corresponded to the ideal expression (28), and the values of $U$ in Eq. (3) and the number of particles $1 \leq N_{p} \leq 10$ were varied independently. The largest number $N_{p}=10$ for $N=10$ corresponds to a Hilbert space dimension of $\left(N+N_{p}-1\right) ! /\left(N_{p}-1\right) ! N !=$ 92378 ; the computational time for the simulation of the spectral transform became unpleasantly long for larger $N_{p}$. The goal was to determine the fidelity $F$ of the spectral transform; i.e. if the initial state was the $k$ th eigenvector of the unperturbed (but interacting) Hamiltonian, then $F$ is the particle density in the $k$ th site of the lattice at the time $t_{s}$.

In practice, one need only consider the fidelity of the $\left|v_{0}\right\rangle \leftrightarrow|0\rangle$ spectral transformation, because this is representative of all the eigenstates. The results of the numerical simulation are shown in Fig. 2. When $U=0.1 J$, the fidelity has dropped to only 0.98 when $N_{p}=N=10$, i.e. at unit lattice filling $\nu \equiv N_{p} / N=1$. The best fit to the data indicates that the fidelity drops as a power law, $F \approx 1-0.023 \nu^{3.3}$. As the on-site interaction strength is increased the fidelity drops; when $\nu=1$ one obtains $F \approx 0.92$ when $U=0.2 J$ and only $F=0.63$ when $U=0.5 \mathrm{~J}$. The best fits in the $U=0.2 \mathrm{~J}$ and $U=0.5 \mathrm{~J}$ cases are $F \approx 1-0.088 \nu^{3.3}$ and $F \approx 1-0.43 \nu^{3.1}$, respectively, both power laws with a similar exponent. Though it is impossible to confidently extrapolate to large system sizes with $N$ and $N_{p}$ comparable to those in a future experiment, the numerical results suggest that the ability to perform a spectral transform to $90 \%$ accuracy or better would require $\nu \lesssim 0.6$ and/or $U \lesssim 0.5 J$. While this value of $U / J$ ensures that the system is in the superfluid regime [57], it is smaller than the value one would generally obtain in the 1D lattice case (cf. Sec. III).

\section{CONCLUSIONS}

The scheme described above to perform the spectral transform with ultracold atoms has assumed that the optical lattice is strictly one-dimensional. While this assumption has simplified the mathematical analysis and the numerical simulations, it is not necessary. In practice, many Bravais lattices are generated by two counterpropagating laser beams in different (not necessarily perpendicular) spatial directions. One could envisage that the lattice beams along each different axis would be suitably prepared so that their intensities were varying quadratically, using either approach discussed in Sec. V. The tunneling amplitudes would then vary along each axis, so that the eigenstates of the lattice (in the noninteracting limit) would correspond approximately to the product of Krawtchouk functions for each direction.

Performing a full three-dimensional spectral transform would not necessarily require a linear potential applied in each direction simultaneously, a task which might be difficult in practice. Instead, one could simply apply the linear ramps for each dimension in sequence, separately cooling the atoms' motions in each spatial degree of freedom. For the cubic lattice for example, eliminating atoms from large site values in one direction would not perturb the atoms in the perpendicular directions, and cooling can be efficiently performed. The presence of interactions will modify this picture somewhat, but if the interaction strength is kept at sufficiently small $(U \lesssim J$ based on the one-dimensional simulations) then this should not present much of a problem.

Besides the obvious benefits of temperature measurement and cooling, the ability to perform a spectral transformation in situ has other possible future applications. For example, the technique could be used to probe the excitation spectrum and heating mechanisms of strongly interacting systems. At any given moment, the interactions could be quickly tuned to approximately zero through the application of Feshbach resonances, and the system's energy spectrum monitored by spectral transform followed by non-destructive imaging; subsequently the system would be returned to its original state by inverting the process. Of course, care would need to be taken to ensure that the ramp on and off of the interactions were slow enough that this process itself would not greatly perturb the system.

As a second example, it is conceivable that the technique could be extended to produce a greater variety of overall phases than the 0 and $\pi$ in the present case (39). In particular, if a suitable choice of tunneling parameters and external potential could be found such that the resulting phases would be of the form $\pi \mathrm{km} / \mathrm{N}$, then the spectral transform would be equivalent to a quantum Fourier transform, a central operation in several efficient quantum algorithms including factoring [58, 59]. In this 
case, each lattice site (out of a total $N=2^{n}$ ) would label a possible state in the $n$-qubit Hilbert space. Though inefficient in terms of resources, this notion could pave the way toward implementation of interesting quantum algorithms using ultracold atoms in optical lattices with minimal external controls.

\section{Acknowledgments}

It is a pleasure to thank M. Oberthaler for stimulating conversations. This work was supported by the Natural
Sciences and Engineering Research Council of Canada and the Canada Foundation for Innovation.
[1] I. Bloch, Nature Phys. 1, 23 (2005).

[2] O. Morsch and M. Oberthaler, Rev. Mod. Phys. 78, 179 (2006).

[3] M. Lewenstein et al., Adv. Phys. 56, 243 (2007).

[4] M. Greiner and M. Fölling, Nature 453, 736 (2008).

[5] I. Bloch, J. Dalibard, and W. Zwerger, Rev. Mod. Phys. 80, 885 (2008).

[6] T. Gericke et al., J. Mod. Opt. 54, 735 (2007).

[7] S. E. Sklarz et al., Phys. Rev. A 66, 053620 (2002).

[8] P. B. Blakie and J. V. Porto, Phys. Rev. A 69, 013603 (2004).

[9] A. M. Rey, G. Pupillo, and J. V. Porto, Phys. Rev. A 73, 023608 (2006).

[10] P. B. Blakie, A. M. Rey, and A. Bezett, Laser Phys. 17, 198 (2007).

[11] S. Schmid et al., New J. Phys. 8, 159 (2006).

[12] R. G. Scott et al., Phys. Rev. A 69, 033605 (2004).

[13] J. Catani et al., Phys. Rev. A 77, 011603(R) (2008).

[14] M. Cramer et al., Phys. Rev. Lett. 100, 140409 (2008).

[15] H. Moritz, T. Stöferle, M. Köhl, and T. Esslinger, Phys. Rev. Lett. 91, 250402.

[16] B. Paredes et al., Nature 429, 277 (2004).

[17] A. Reischl, K. P. Schmidt, and G. S. Uhrig, Phys. Rev. A 72, 063609 (2005).

[18] J. I. Cirac and P. Zoller, Science 301, 176 (2003).

[19] G.-D. Lin, Wei Zhang, and L.-M. Duan, Phys. Rev. A 77, 043626 (2008).

[20] R. B. Diener, Q. Zhou, H. Zhai, and T. L. Ho, Phys. Rev. Lett. 98, 180404 (2007).

[21] F. Gerbier, S. Fölling, A. Widera, and I. Bloch, arXiv:cond-mat/0701420v1 (2007).

[22] G. Pupillo, C. J. Williams, and N. V. Prokof'ev, Phys. Rev. A 73, 013408 (2006).

[23] B. Capogrosso-Sansone, E. Kozik, N. Prokof'ev, and B. Svistunov, Phys. Rev. A 75, 013619 (2007).

[24] A. Griessner et al., Phys. Rev. Lett. 97, 220403 (2006).

[25] M. Popp, J.-J. Garcia-Ripoll, K. G. H. Vollbrecht, and J. I. Cirac, New. J. Phys. 8, 164 (2006).

[26] C. J. Pethick and H. Smith, 'Bose-Einstein Condensation in Dilute Gases,' Cambridge University Press, 2001.

[27] M. Krawtchouk, Comptes Rendus 189, 620 (1929).

[28] R. Álvarez-Nodarse and J. L. Cardoso, J. Differ. Equ. Appl. 11, 829 (2005).

[29] H.-J. Miesner et al., Science 279, 1005 (1998).

[30] M. Greiner et al., Appl. Phys. B 73, 769 (2001).

[31] M. Greiner et al., Nature 415, 39 (2002).
[32] M. L. Chiofalo and M. P. Tosi, Phys. Lett. A 268, 406 (2000).

[33] E. G. M. van Kempen, S. J. J. M. F. Kokkelmans, D. J. Heinzen and B. J. Verhaar, Phys. Rev. Lett. 88, 093201 (2002).

[34] K. E. Strecker, G. B. Partridge, A. G. Truscott, and R. G. Hulet, Nature 417, 150 (2002).

[35] T. P. Meyrath et al., Phys. Rev. A 71, 041604 (2005).

[36] K. D. Nelson, X. Li, and D. S. Weiss, Nature Phys. 3, 556 (2007).

[37] D. Jaksch et al., Phys. Rev. Lett. 81, 3108 (1998).

[38] M. P. A. Fisher, P. B. Weichmann, G. Grinstein, and D. S. Fisher, Phys. Rev. B 40, 546 (1989).

[39] C. J. Hamer and J. B. Kogut, Phys. Rev. B 20, 3859 (1979).

[40] H. H. Roomany and H. W. Wyld, Phys. Rev. D 21, 3341 (1980).

[41] N. Shenvi, J. Kempe, and K. B. Whaley, Phys. Rev. A 67, 052307 (2003).

[42] M. Christandl, N. Datta, A. Ekert, and A. J. Landahl, Phys. Rev. Lett. 92, 187902 (2004).

[43] M. Christandl et al., Phys. Rev. A 71, 032312 (2005).

[44] D. L. Feder, Phys. Rev. Lett. 97, 180502 (2006).

[45] J. Kempe. Contemp. Phys. 44, 307 (2003).

[46] A. Ambainis, Int. J. Quantum Inform. 1, 507 (2003).

[47] J. Fitzsimons and J. Twamley, Phys. Rev. Lett. 97, 090502 (2006).

[48] V. Kostak, G. M. Nikolopoulos, I. Jex, Phys. Rev. A 75, 042319 (2007).

[49] I. D'Amico, B. W. Lovett, and T. P. Spiller, Phys. Rev. A 76, 030302 (2007).

[50] T. Hakioğlu and K. B. Wolf, J. Phys A 33, 3313 (2000).

[51] Numerical Recipes: The Art of Scientific Computing, 3rd Ed., Cambridge University Press, 2007. W. H. Press, S. A. Teukolsky, W. T. Vetterling, and B. P. Flannery, p. 185 .

[52] P. Feinsilver and J. Kocik, Contemporary Mathematics 287, 83 (2001).

[53] P. Feinsilver and J. Kocik, Recent Advances in Applied Probability, R. Baeza-Yates et al. (eds), Springer Verlag, 2005, pp. 115-143.

[54] N. M. Atakishiyev and K. B. Wolf, J. Opt. Soc. Am. A 14, 1467 (1997).

[55] P.-T. Yap, R. Paramesran, S.-H. Ong, IEEE Trans. Image Proc. 12, 1367 (2003).

[56] M. Fattori et al., Phys. Rev. Lett. 100, 080405 (2008). 
[57] T. Stöferle et al., Phys. Rev. Lett. 92, 130403 (2004).

[58] D. E. Browne, New J. Phys. 9, 146 (2007).

[59] N. Yoran, N. J. Short, Phys. Rev. A 76, 060302 (2007). 